\title{
A Method for Ranking Battlefield Damaged Equipment Repairs Based on Projection Pursuit Method
}

\author{
Wang Xiao-yan,Shi Tao, Dong Yu-cai, Zheng Su-wen,Zheng Dong-yun,Pan \\ Xue -qin,Du Jian,Jin Qi
}

(Department of Fundamental Courses, Academy of Armored Force Engineering, Beijing, 100072, China)

Keywords: projection pursuit method; battlefield damaged equipment; ranking repairs.

\begin{abstract}
The key impact indicators of battlefield damage equipment repair order are transformed into one-dimensional projection values with the method of projection pursuit. The repair order of battlefield damage equipment is got and weights of all key performance indicators are clear. The example is applied to explain application effect of this method. The experiment shows that the method is easy to decision-making, strong objectivity, applicability and operability, has great significance to enhance the combat effectiveness of troops.
\end{abstract}

\section{Introduction}

Battlefield repair of the equipment is very important in technical support work in wartime, which is an important factor to maintain and restore the combat effectiveness of troops. It can effectively reduce the loss rate of equipment and increase the number of equipment deployed to improve the combat effectiveness[1,2,3]. Battlefield repairs have great difficulty and some limitations because of the particular environment and conditions of wartime. Improving the ability of battlefield equipment repairs is the most effective measure and fundamental way to reduce loss rate of equipment. Countermeasures to solve this difficulty is reasonable arrangement the repair order of battlefield damaged equipment under the premise of repair power in wartime, in addition to appropriately increase the strength of repair preparation. It can improve the overall security effectiveness of equipment, and ensure the troops sustained combat and win on battlefield.

The sorting or preferred of battlefield damaged equipment repairs not only need determining scientific and rational evaluation system, but also objective and rational allocation of weights of evaluation indexes. At present, commonly used methods are AHP, fuzzy comprehensive evaluation method, multi-criteria method, value engineering, and decision-making method based on entropy weight multi-objective and so on[4,5]. These methods have their own advantages, but they have a certain degree of subjective and arbitrary on subjective level of knowledge of the importance of all indicators and making the weights are uncertainties. This paper introduces a method for ranking battlefield damaged equipment repairs based on projection pursuit method. The method is objective, which can better reflect the relative importance of each indicator and make equipment repair sorting more reasonable. Finally, an example of radar equipment is introduced to show the method is scientific and reliable on battlefield damaged equipment repairs sorting.

\section{Projection pursuit method}

The projection pursuit method [6] is proposed by Friedman and Turkey in 1974. It is a clustering and classification method for exploratory analysis, and also for uncertainty analysis. Its essence is to observe data from a different angle, and find the optimal projection method which can greatest extent to reflect data characteristics and fully exploit data information. The projection pursuit method is an effective technique for dimensionality reduction. It is suitable for high-dimensional, nonlinear, non-normal analysis and processing. Its evaluation results consistent with the actual , and it has been widely used in water quality assessment, disaster assessment, industrial economy, enterprise competitiveness and so on [7-11]. 
The principle of projection pursuit method is as follows.

(1) Normalized processing of sample evaluation value, which is calculated as

$$
x(i, j)=\frac{x^{*}(i, j)-x \min (j)}{x \max (j)-x \min (j)}
$$

$x^{*}(i, j), i=1, \cdots, n ; j=1, \cdots, p$ is value for the $\mathrm{i}$-th sample of the $\mathrm{j}$ - th evaluation. $n, p$ are respectively the number of samples and the number of evaluation indicators. $\max (j), \min (j)$ are respectively the maximum and minimum value of the $j$ - th evaluation index.

(2) Construct projection objective function $Q(a)$.

A P-dimensional data $x(i, j), j=1, \cdots, p$ are become to one-dimensional projection value $z(i)$ which $a=(a(1), a(2), \cdots, a(p))$ are the projection direction. The formula is

$$
z(i)=\sum_{i=1}^{n} a(j) x(i, j), \quad i=1, \cdots, p
$$

Where $a_{\text {is }}$ the unit length vector.

The distribution characteristics of the projection values are that local projection points are as possible intensive, and best to condense into a number of point group, while the projection point group spread out as much as possible. Based on these, the projection index function can be constructed as

$$
Q(a)=S_{z} D_{z}
$$

$S_{z}$ is the standard deviation of projection value $z(i), D_{z}$ is the local density of projection value $z(i)$. The formulas are

$$
\begin{gathered}
S_{z}=\sqrt{\sum_{i=1}^{n} z(i)-\bar{z} /(n-1)} \\
D_{z}=\sum_{i=1}^{n} \sum_{j=1}^{n}\left(R-r_{i j}\right) u\left(R-r_{i j}\right) \\
r_{i j}=|z(i)-z(j)|
\end{gathered}
$$

$\bar{z}$ is the mean of $z(i), i=1, \cdots, n . R$ is the window radius of the local density, $u_{t}$ is the unit step function, When $t=\left(R-r_{i j}\right) \geq 0$, its function value is 1 ; When $t<0$, its function value is 0 . The window radius $R$ of the local density neither be too small avoiding the average number of projection point in window is too little and moving average deviation is too large nor too high with the increasing of the number of indicators increased. It can be $0.1 S_{z}, 0.01 S_{z}, 0.001 S_{z}$ and so on.

(3) Assessment of the best projection direction by solving the maximum of projection index function. It reveals certain characteristic structure of high dimensional data. The formula is

$$
\begin{aligned}
& \max Q(' a)=S_{z} D_{z} \\
& \text { s.t. } \sum_{j=1}^{p} a^{2}(j)=1, \quad a(j) \geq 0
\end{aligned}
$$

The above equation is a complex nonlinear optimization problems with optimization variable $a(j), j=1, \cdots, p$. The optimization problems of projection index function is the key to success of the application of projection pursuit method. Its calculation is quite large. The paper uses simplex method to solve multi-constrained global optimization problem.

(4)The projection values $z(i)$ of evaluation samples are got from the formula $z(i)=\sum_{i=1}^{n} a(j) x(i, j)$. Ranking according to $z(i)$ from big to small can evaluate evaluation indexes of sample set . The 
optimal projection direction vector $a^{*}$ reflects the different degree of importance of each factor and satisfies $a^{* 2}=1$, so it is served as weights of influence factors .

\section{Battlefield Damaged Equipment Sorting of Radar Network}

Information warfare increasingly dependents on intelligence information detected by early warning radar. Intelligence information is a slight delay, it will lose the best time to combat, thus it affects the process and even the outcome of wars. Therefore, the ranking of radar network battlefield damaged equipment repairs not only considers their detection efficiency ratio (ratio of detection airspace of single-radar and the radar network), necessary equipment and persons, more importantly consider the substitutability of their combat missions and repair time .

Table 1 evaluation form of radar network battlefield damaged equipment repair sorting

\begin{tabular}{|c|c|c|c|c|c|c|}
\hline $\begin{array}{c}\text { Battle } \\
\text { damage } \\
\text { equipment }\end{array}$ & $\begin{array}{c}\text { Substitut } \\
\text { e index of } \\
\text { combat } \\
\text { missions } x_{1}\end{array}$ & $\begin{array}{c}\text { Detection } \\
\text { efficiency } \\
\text { ratio of radar } \\
\text { network } \% x_{2}\end{array}$ & $\begin{array}{c}\text { Necessary } \\
\text { equipment of } \\
\text { repair } \\
x_{3} \text { (item) }\end{array}$ & $\begin{array}{c}\text { Necessary } \\
\text { personnel of } \\
\text { repair } \\
x_{4} \text { (person) }\end{array}$ & $\begin{array}{c}\text { Necessary } \\
\text { time of repair } \\
x_{5} \text { (hour) }\end{array}$ \\
\hline Unit & Model & 6 & 9 & 8 & 4 & 3.5 \\
\hline 3Station & LLQ $\times \times 1$ & 13 & 5 & 5 & 4 \\
\hline 6Station & LLQ $\times \times 2$ & 5 & 5 & 6 & 5 & 2.5 \\
\hline 7Station & LLQ $\times \times 3$ & 9 & 15 & 4 & 6 & 4.5 \\
\hline \multicolumn{2}{|c|}{ Indicator types } & 0 & 1 & 0 & 0 & 0 \\
\hline
\end{tabular}

It is assumed that four radar equipments belong to different radar station in a radar network appear damage at the same time[5]. The priority of radar network battlefield damaged equipment repairs are evaluated by five indicators. The evaluation data of radar network battlefield damaged equipment determined by equipment information and battlefield damaged assessment level is shown in Table 1.There are two kinds of indicator types in the table. "1" indicates that the index is efficiency, and "0" indicates that the index is cost type.

Density threshold value is $0.1 S_{z}$, and the best projection direction and weight coefficients of evaluation index are in Table 2.

Table 2 The best projection direction and weighting coefficient table of evaluation index

\begin{tabular}{|l|c|c|}
\hline evaluation index & projection direction & weight coefficient \\
\hline Substitute index of combat missions & 0.6088 & 0.3707 \\
\hline Detection efficiency ratio of radar network & 0.7467 & 0.5575 \\
\hline Necessary equipment of repair & 0.1548 & 0.0240 \\
\hline Necessary personnel of repair & 0.1186 & 0.0140 \\
\hline Necessary time of repair & 0.1839 & 0.0338 \\
\hline
\end{tabular}

From table 2 ,we can see that Substitute index of combat missions and detection efficiency ratio of radar network are the most important indicators, weight of other indicators are less than 0.1 .

The projection values and sorting of radar network battlefield damaged equipment are shown in Table 3.

Table 3 The projection values and sorting of radar network battlefield damaged equipment

\begin{tabular}{|c|c|c|c|}
\hline \multicolumn{2}{|c|}{ Battlefield damaged equipment } & Projectio \\
Unit & Model & $\begin{array}{c}\text { Sortin } \\
\text { values }\end{array}$ & \\
\hline 1 Station & LLQ $\times \times 1$ & 1.7382 & 3 \\
\hline 3 Station & LLQ $\times \times 2$ & 15.5876 & 2 \\
\hline 6 Station & LLQ $\times \times 3$ & 7.2999 & 4 \\
\hline 7 Station & LLQ $\times \times 4$ & 18.8516 & 1 \\
\hline
\end{tabular}


If the sort uses the method of fuzzy comprehensive evaluation sorting decision-making proposed by literature [12], experts give scores to importance level of the individual indicators ,and then calculate the weight of each indicator, it has a certain degree of operability, and its results are also consistent with this article. If the sort uses the method proposed by literature [5], subjective weights are combined with objective entropy weight, the results obtained are consistent with this article. Therefore, the method is reasonable. Projection pursuit method is more objective to evaluation objects, sort result of battlefield damage equipment is more reasonable and scientific compared with other methods.

\section{Conclusion}

Battlefield repair is an important factor to maintain and restore combat effectiveness of equipment troops. Reasonable arrangements for the repair not only repair more damage equipments in a limit time , improve the work efficiency of the equipment repair system, but also give priority to protecting the vital equipments and improve effectiveness of battlefield repair. Projection pursuit method proposed by the papar can scientific sort to battlefield damaged equipment. The method is easy to decision-making, has strong objectivity, applicability and operability, and great significance to improve combat effectiveness of equipment troops. .It is achieved by computer programming in practical applications. We can first establish repair database of equipment in order to improve the timeliness of command and decision in wartime .

\section{References}

[1] Li Chuan-liang, Battlefield Repair of equipment[J]. Journal of Sichuan Ordnance, 2008 (4) :102-103.

[2] He Ji,Zhang Shi-zhong,Zhou Le-rong,etc. Study on Task Assignment Model of Wartime Equipment Maintenance[J]. Value Engineering,2010,29(35) : 295-296..

[3] Xu Lei,Wang Wen-feng,Yang Jian-jun..Tasks Assigned Model of Different Emergence Battlefield Damage [J]. CH INA SH IPREPA IR, 2008(B06): 37-39,42

[4] Yan Bing-bin, Xu Hang, Shi Quan. Decision-making Model for Ranking Battlefield Damaged Equipment Repairs Based on Multi- criteria[J]. Journal of Ordnance Engineering College. 2008, 20(5): 19-21,25.

[5] Chen Yong-jian , Guo Yi-zhong , Jiang Zhi-min,etc. A Method for Ranking Battlefield Damaged Equipment Repairs of Radar Net Based on Entropy Weight Multi-objective Decision Makin[J]. Journal of Ordnance Engineering College, 2008， 20(5): 19-21,25

[6] Friedman JH, Tukey JW.A. Projection Pursuit Algorithm for Exploratory Data Analysis[J]. IEEE Trans Compute, 1974.23(1):881-890.

[7] Zhang Lian-peng, Liu Qing-huo, Liu Guo-lin,etc. Multi-directional Projection Pursuit and Feature Extraction of Hyperspectral Remote Sensing Image[J] . Chinese Science Abstracts, 2007, 13(12): 97-97

[8] Gong Yan,Shu ning.Study on Dimensionality Reduction Method of Hyperspectral Images Based on Successive Projection Pursuit [J].Geomatics Information and Engineering 2007, 32(3): 1-3.

[9] Wang Xiu-peng,Zhang Hong-cai,Zhao Yong-qiang,etc. Fusion algorithm of hyperspectral image feature based on Projection Pursuit[J].Computer Measurement \& Control,2006, 14(11) : 1539-1541. 
[10] Song Hao-feng, Fu Qiang,LI Chune-bo,etc. Parametric Projection Pursuit Model Applied in The Optimization of Water Diversion Routes[J].Northeast Water Resources and Hydropower, 2006, 24(5): 6-8.

[11] Wang Zhi-xing,Li Cheng-zhen,Chen Gang. Projection Pursuit Regression Model of Ice Conditions Forecast[J].Jounnal of Natural Disasters, 2009, 18(5): 174-177.

[12] Deng Hui-yong,Ma Ji-sheng,Wang Yan,etc. Assessment of Artillery Quality Based on Fuzzy Comprehensive Evaluation[J].Journal of Gun Launch \& Control, 2008(1): 20-22. 